\title{
Planar Bipedal Walking with Foot Rotation
}

\author{
Jun Ho Choi and J. W. Grizzle
}

\begin{abstract}
This paper addresses the key problem of walking with both fully actuated and underactuated phases. The studied robot is planar, bipedal, and fully actuated in the sense that it has feet with revolute, actuated ankles. The desired walking motion is assumed to consist of three successive phases: a fully-actuated phase where the stance foot is flat on the ground, an underactuated phase where the stance heel lifts from the ground and the stance foot rotates about the toe, and an instantaneous double support phase where leg exchange takes place. The main contribution of the paper is to provide a provably asymptotically stabilizing controller that integrates the fully-actuated and underactuated phases of walking. By comparison, existing humanoid robots, such as Asimo and Qrio, use only the fully-actuated phase (i.e., they only execute flat-footed walking), or RABBIT, which uses only the underactuated phase (i.e., it has no feet, and hence walks as if on stilts). The controller proposed here is organized around the hybrid zero dynamics of Westervelt et al. (2003) in order that the stability analysis of the closed-loop system may be reduced to a one-dimensional Poincaré map that can be computed in closed form.
\end{abstract}

\section{INTRODUCTION}

Over the past seven years, several remarkably anthropomorphic robots have been constructed. Specifically, we have in mind the well known Honda Robot, Asimo [1], [2], Sony's biped, SDR-4X [3], and Jogging Johnnie at the University of Munich [4], [5]. The Honda and Sony robots are especially noteworthy for their autonomy, while Jogging Johnnie's construction appears to be particularly light and graceful (1.8 m tall, $40 \mathrm{Kg}$ mass, which includes sensors, actuators and control hardware, though power is supplied through a cable). Each of these robot's control systems is organized around a high-level trajectory generator for the individual joints of the robot, combined with lowlevel servoing to ensure trajectory tracking. There are some differences in how the low-level servoing is implementedHonda uses PD control [2], Jogging Johnnie uses feedback linearization [5], while Sony has revealed little about their algorithms - but these differences are fairly insignificant. In each case, the overall "stability" of the robot's motion has been "ensured" by the zero moment point (ZMP) criterion (see Figure 1), and consequently, these robots literally walk flat footed.

A stability analysis of a flat-footed walking gait for a five-link biped with an actuated ankle was carried out numerically in [6], [7], using the Poincaré return map. The control law used feedback linearization to maintain the robot's posture and advance the swing leg; trajectory

This work was supported by NSF grant ECS-0322395.

Jun Ho Choi and J. W. Grizzle are with the Department of Electrical Engineering and Computer Science, University of Michigan, Ann Arbor, Michigan 48109-2122, USA \{junhoc, grizzle\}@umich.edu

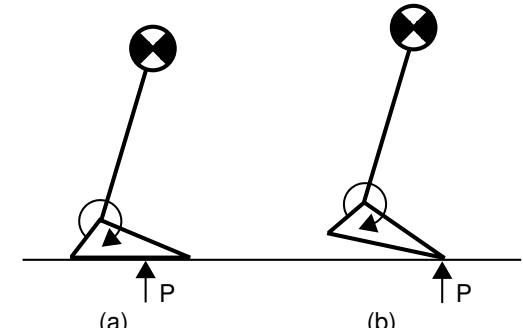

Fig. 1. The ZMP (Zero Moment Point) principle in a nutshell. Idealize a robot in single support as a planar inverted pendulum attached to a base consisting of a foot with torque applied at the ankle and all other joints are independently actuated. Assume adequate friction so that the foot is not sliding. In (a), the robot's nominal trajectory has been planned so that the center of pressure of the forces on the foot, $P$, remains strictly within the interior of the footprint. In this case, the foot will not rotate (i.e, the foot is acting as a base, as in a normal robotic manipulator), and thus the system is fully actuated. It follows that small deviations from the planned trajectory can be attenuated via feedback control, proving stabilizability of the walking motion. In case (b), however, the center of pressure has moved to the toe, allowing the foot to rotate. The system is now underactuated (two degrees of freedom and one actuator), and designing a stabilizing controller is nontrivial, especially when impact events are taken into account. The ZMP principle says to design trajectories so that case (a) holds; i.e., walk flat footed. Humans, even with prosthetic legs, use foot rotation to decrease energy loss at impact.

tracking was only used in the limited sense that the horizontal component of the center of mass was commanded to advance at a constant rate. The unilateral constraints due to foot contact were carefully presented. Motivated by energy efficiency, elegant work in [8], [9] has shown how to realize a passive walking gait in a fully actuated biped robot walking on a flat surface. Stability of the resulting walking motion has been rigorously established. The main drawback, however, is that the assumption of full actuation once again restricts the foot motion to flat-footed walking.

For walking gaits that include foot rotation, various ad hoc control solutions have been proposed in the literature [10], [11], [12], [13], [14], [15], but none of them can guarantee stability in the presence of the underactuation that occurs during heel roll or toe roll. Our previous work on point feet [16], [17], [18], [19], [20] ideally positions us to handle this underactuation; indeed, conceptually, a point foot corresponds to continuous rotation about the toe throughout the entire stance phase (e.g., walking like a ballerina or as if on stilts). Work in [21] shows that plantarflexion of the ankle, which initiates heel rise and toe roll, is the most efficient method to reduce energy loss at the subsequent impact of the swing leg. This motion is also necessary for the esthetics of mechanical walking.

In this paper, we extend our analysis of walking with point feet to design a controller that provides asymptotically 


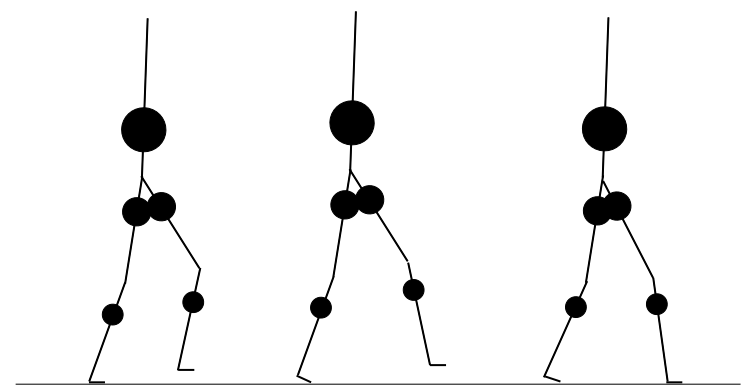

Fig. 2. The three phases of walking modelled in this paper: fully-actuated phase where the stance foot is flat on the ground, underactuated phase where the stance heel rises from the ground and the stance foot rotates about the toe, and double support phase where the swing foot impacts the ground.

stable walking with an anthropomorphic foot motion. In particular, the achieved walking motion consists of three successive phases ${ }^{1}$ : a fully-actuated phase where the stance foot is flat on the ground, an underactuated phase where the stance heel lifts from the ground and the stance foot rotates about the toe, and an instantaneous double support phase where leg exchange takes place, see Figure 2.

\section{Robot ModeL}

The robot considered in this paper is bipedal and planar with $N \geq 4$ rigid links connected by ideal (frictionless) revolute joints to form a tree structure (no closed kinematic chains). It is assumed to have two identical open chains called "legs" that are connected at a point called the "hips." The link at the extremity of each leg is called a "foot" and the joint between the foot and the remainder of the leg is called an "ankle." The feet are assumed to be "forward facing." The forward end of each foot is called a "toe" and the back end is called a "heel." Each revolute joint is assumed to be independently actuated. It is assumed that walking consists of three successive phases, a fully-actuated phase, an underactuated phase, and a double support phase, see Figure 2 and 3. The detailed assumptions for the robot and the gait are listed in Appendix A. A representative robot is shown in Figure 4 along with a coordinate convention.

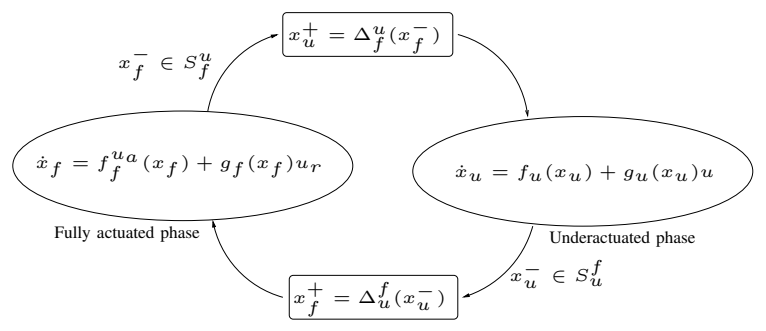

Fig. 3. Diagram of system.

\footnotetext{
${ }^{1}$ For simplicity, heel strike with a subsequent heel roll is not addressed. It can be handled in the same manner as toe roll.
}
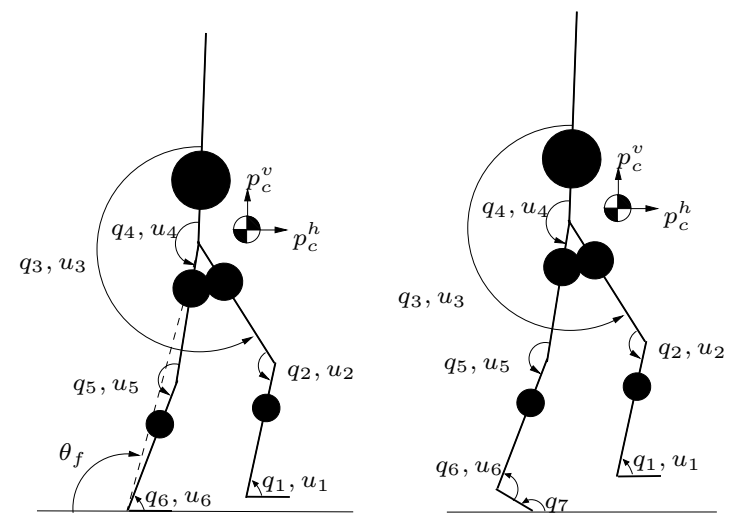

Fig. 4. Model of a 7-link robot with coordinate convention. In general, for $N$-link robot, it is assumed that $q_{N-1}$ is the angle between the foot and the femur and $q_{N}$ is the angle between the ground and the foot.

\section{A. Underactuated phase}

The underactuated phase is when the stance heel of the robot rises from the ground and the robot begins to roll over the stance toe; this condition is characterized by the foot rotation indicator (FRI) point of [22] being strictly in front of the stance foot. The stance toe is assumed to act as a pivot; this condition is characterized by the forces at the toe lying within the allowed friction cone. Both of these conditions (i.e., foot rotation and non-slip) are constraints that must be imposed in the final controller design phase, as in $[17, \mathrm{Sec}$. VI].

Since there is no actuation between the stance toe and the ground, the dynamics of the robot in this phase is equivalent to an $N$-DOF robot with unactuated point feet and identical legs, as treated in [17]. Define the generalized coordinates as $q_{u}=\left(q_{1}, \cdots, q_{N}\right)^{T} \in \mathcal{Q}_{u}$, where $\mathcal{Q}_{u}$ is a simply connected open subset of $\mathbb{R}^{N}$. The dynamics are obtained using the method of Lagrange, yielding

$$
D_{u} \ddot{q}_{u}+C_{u} \dot{q}_{u}+G_{u}=B_{u} u,
$$

where $u=\left(u_{1}, \cdots, u_{N-1}\right)^{T}$ is the vector of torques applied at the joints. The dynamic equation in state-variable form is expressed as $\dot{x}_{u}=f_{u}\left(x_{u}\right)+g_{u}\left(x_{u}\right) u$.

\section{B. Fully-actuated phase model}

During the fully-actuated phase, the stance foot is assumed to remain flat on the ground without slipping. The ankle of the stance leg is assumed to act as an actuated pivot. Since the stance foot is motionless during this phase, the dynamics of the robot during the fully-actuated phase is equivalent to an $N-1$ DOF robot without the stance foot and with actuation at the stance ankle, as studied in [23]. Let $q_{f}=\left(q_{1}, \cdots, q_{N-1}\right)^{T} \in \mathcal{Q}_{f}$ be the configuration variables, where $q_{1}, \cdots, q_{N-2}$ denote the relative angles of the joints except the stance ankle, $q_{N-1}$ denotes the angle of the ankle joint, and $\mathcal{Q}_{f}$ is a simply connected open subset of $\mathbb{R}^{N-1}$, see Figure 4 . Note that because the stance foot remains on the ground, $q_{N-1}$ is now an absolute angle (i.e., it is referenced to the world frame). 
The dynamics for the fully-actuated phase are derived using the method of Lagrange, yielding a model of the form

$$
D_{f}\left(q_{f}\right) \ddot{q}_{f}+C_{f}\left(q_{f}, \dot{q}_{f}\right) \dot{q}_{f}+G_{f}\left(q_{f}\right)=B_{f 1} u_{R}+B_{f 2} u_{A},
$$

where $\dot{q}_{f}$ are the velocities, $u_{R}=\left(u_{1}, \cdots, u_{N-2}\right)^{T}$ are the inputs applied at the joints except the ankle joint, and $u_{A}=u_{N-1}$ is the input at the ankle joint. The state is taken as $x_{f}=\left(q_{f} ; \dot{q}_{f}\right) \in T \mathcal{Q}_{f}$ and the dynamic equation is given by ${ }^{2}$

$$
\begin{aligned}
\dot{x}_{f}= & {\left[\begin{array}{c}
\dot{q}_{f} \\
D_{f}^{-1}\left(-C_{f} \dot{q}_{f}-G_{f}+B_{f 2} u_{A}\right)
\end{array}\right] } \\
& +\left[\begin{array}{c}
0 \\
D_{f}^{-1} B_{f 1} u_{R}
\end{array}\right] \\
=: & f_{f}^{u_{A}}\left(x_{f}\right)+g_{f}\left(x_{f}\right) u_{R} .
\end{aligned}
$$

Note that, to satisfy the condition that the stance foot is flat on the ground, the FRI point needs to be kept strictly within the support region. This constraint must be imposed on designing the controller as in [17, Sec. V].

\section{Double support phase}

During the double support phase, the swing foot impacts the ground. It is assumed that the swing foot is parallel to the ground at impact. It is also assumed that the feet are arc shaped so that the only contact points with the ground are the heel and the toe. Due to the impacts, impulsive forces are applied at the toe and the heel, which cause discontinuous changes in the velocities; however, the position states are assumed to remain continuous [24].

Representing the double support phase requires an $\mathrm{N}+2$ DOF model (e.g. N DOF for the joints and 2 DOF for the position of the center of mass). Adding Cartesian coordinates, $\left(p_{c}^{h}, p_{c}^{v}\right)$, to the center of mass of the robot gives $q_{d}=\left(q_{u} ; p_{c}^{h} ; p_{c}^{v}\right)$ and $\dot{q}_{d}=\left(\dot{q}_{u} ; \dot{p}_{c}^{h} ; \dot{p}_{c}^{v}\right)$, see Figure 4 . Let $\Upsilon_{h}\left(q_{d}\right)$ and $\Upsilon_{t}\left(q_{d}\right)$ denote the Cartesian coordinates of the swing heel and the swing toe, respectively. The method of Lagrange yields the dynamics for the double support phase as follows:

$$
\begin{array}{r}
D_{d}\left(q_{d}\right) \ddot{q}_{d}+C_{d}\left(q_{d}, \dot{q}_{d}\right) \dot{q}_{d}+G_{d}\left(q_{d}\right) \\
=B_{d} u+E_{d}^{h} \delta F_{h}+E_{d}^{t} \delta F_{t},
\end{array}
$$

where $u=\left(u_{R}^{T}, u_{A}\right)^{T}, E_{d}^{h}=\left(\frac{\partial \Upsilon_{h}\left(q_{d}\right)}{\partial q_{d}}\right)^{T}, E_{d}^{t}=$ $\left(\frac{\partial \Upsilon_{t}\left(q_{d}\right)}{\partial q_{d}}\right)^{T}, \delta F_{h}$ denotes the impulsive ground reaction force at the swing heel, and $\delta F_{t}$ denotes the impulsive ground reaction force at the swing toe.

Under the hypothesis IH6 (the actuators not being impulsive) and IH1 (which is the stance foot neither rebounds

\footnotetext{
${ }^{2}$ Note that the ankle torque is included in $f_{Z_{f}}^{u_{A}}\left(x_{f}\right)$; the reason for this will be clear in Section III.
}

nor slips), following the procedure in [16] gives

$$
\begin{aligned}
x_{f}^{+} & =\left[\begin{array}{cc}
{\left[\begin{array}{cc}
R & 0
\end{array}\right] q_{d}^{-}} \\
{\left[\begin{array}{ll}
R & 0
\end{array}\right] \Pi\left[\begin{array}{c}
D_{d} \dot{q}^{-} \\
0
\end{array}\right]}
\end{array}\right] \\
& =\Delta_{u}^{f}\left(x_{d}^{-}\right),
\end{aligned}
$$

where $R$ is a relabeling matrix and

$$
\Pi=\left[\begin{array}{ccc}
D_{d} & -E_{d}^{1} & -E_{d}^{2} \\
E_{d}^{1 T} & 0 & 0 \\
E_{d}^{2 T} & 0 & 0
\end{array}\right]^{-1}
$$

\section{Foot Rotation, or Transition from Full Actuation to Underactuation}

The transition from a flat foot to rotation about the toe can be initiated by causing the angular acceleration about the stance toe to become negative. To characterize the motion of the stance foot or equivalently, when the robot transitions from full actuation-foot flat on the ground-to underactuation-foot rotates about the toe, the foot rotation indicator (FRI) point of Goswami is used [22, eq. (6)].

By enforcing that the FRI point is strictly in front of the end of the foot, the transition is initiated. If torque discontinuities $^{3}$ are allowed-as they are assumed to be in this paper-when to allow foot rotation becomes a control decision. Here, in view of simplifying the analysis of periodic orbits in Section IV, the transition is assumed to occur at a fixed point in the fully-actuated phase. Hence, $H_{f}^{u}=\theta_{f}\left(q_{f}\right)-\theta_{f_{f}}^{-}$, where $\theta_{f}(q)$ is the angle of the hips with respect to the stance ankle (see Figure 4 ) and $\theta_{f}^{-}$is a constant to be determined.

The positions and the velocities are assumed to remain continuous even with the discontinuous torque. The ensuing initial value of the underactuated phase, $x_{u}^{+}$, is defined so as to achieve continuity in the position and velocity variables:

$$
x_{u}^{+}=\left[\begin{array}{c}
q_{u}^{+} \\
\dot{q}_{u}^{+}
\end{array}\right]=\left[\begin{array}{c}
q_{f}^{-} \\
\pi \\
\dot{q}_{f}^{-} \\
0
\end{array}\right]=: \Delta_{f}^{u}\left(x_{f}^{-}\right) .
$$

Continuity of the torques is not imposed, and hence neither is continuity of the accelerations. It is assumed that the control law in the underactuated phase will be designed to achieve the FRI point in front of the toe.

\section{E. Overall Hybrid Model}

As in [25], the overall model can be expressed as a nonlinear hybrid system containing two state manifolds

\footnotetext{
${ }^{3}$ This is a modeling decision. In practice, the torque is continuous due to actuator dynamics. It is assumed here that the actuator time constant is small enough that it need not be modeled.
} 
(called "charts" in [26]):

$$
\begin{gathered}
\Sigma_{f}:\left\{\begin{aligned}
\mathcal{X}_{f} & =T \mathcal{Q}_{f} \\
\mathcal{F}_{f}: \dot{x}_{f} & =f_{f}^{u_{A}}\left(x_{f}\right)+g_{f}\left(x_{f}\right) u_{R} \\
S_{f}^{u} & =\left\{x_{f} \in T \mathcal{Q}_{f} \mid H_{f}^{u}\left(x_{f}\right)=0\right\} \\
\mathcal{T}_{f}^{u}: x_{u}^{+} & =\Delta_{f}^{u}\left(x_{u}^{-}\right)
\end{aligned}\right. \\
\Sigma_{u}:\left\{\begin{aligned}
\mathcal{X}_{u} & =T \mathcal{Q}_{u} \\
\mathcal{F}_{u}: \dot{x}_{u} & =f_{u}\left(x_{u}\right)+g_{u}\left(x_{u}\right) u \\
S_{u}^{f} & =\left\{x_{u} \in T \mathcal{Q}_{u} \mid H_{u}^{f}\left(x_{u}\right)=0\right\} \\
\mathcal{T}_{u}^{f}: x_{f}^{+} & =\Delta_{u}^{f}\left(x_{u}^{-}\right)
\end{aligned}\right.
\end{gathered}
$$

where, for example, $\mathcal{F}_{f}$ is the flow on state manifold $\mathcal{X}_{f}$, $S_{f}^{u}$ is the switching hyper-surface for transitions between $\mathcal{X}_{f}$ and $\mathcal{X}_{u}, \mathcal{T}_{f}^{u}: S_{f}^{u} \rightarrow \mathcal{X}_{u}$ is the transition function applied when $x_{f} \in S_{f}^{s}$.

The transition from the underactuated phase to the fullyactuated phase occurs when the swing foot impacts the ground. Hence, $H_{u}^{f}\left(x_{u}\right)=\Upsilon_{h}^{v}\left(x_{u}\right)$, where $\Upsilon_{h}^{v}\left(x_{u}\right)$ denotes the vertical coordinate of the swing heel.

\section{Control Law Development Based on the HYBRID ZERO DYNAMICS}

In a certain sense, the basic idea of the control law design is quite straightforward. The greatest difficulties in the control design and analysis involve the underactuated phase of the motion. Following the development in [17], [19], we use the method of virtual constraints to create a two-dimensional zero dynamics manifold $Z_{u}$ in the $2 N$ dimensional state space of the underactuated phase. This requires the use of the full complement of $N-1$ actuators on the robot. In the fully-actuated phase, we have one less degree of freedom because the stance foot is motionless and flat on the ground. Consequently, we use $N-2$ actuators-all actuators except the ankle of the stance foot-to create a two-dimensional zero dynamics manifold $Z_{f}$ - that is compatible with $Z_{u}$-in the sense that the following invariance conditions hold: $\Delta_{f}^{u}\left(S_{f}^{u} \cap Z_{f}\right) \subset Z_{u}$ and $\Delta_{u}^{f}\left(S_{u}^{f} \cap Z_{u}\right) \subset Z_{f}$. The actuation authority at the ankle is subsequently employed for stability and efficiency augmentation, and for enforcing the non-rotation of the foot. The invariance conditions guarantee the existence of a hybrid zero dynamics for the closed-loop hybrid model. The techniques in [17] are then extended to compute the Poincaré map of the closed-loop system in closed form. Precise stability conditions then follow.

In the following, the key elements of this program are outlined. Due to space limitations, a more complete analysis, which is actually a simplification ${ }^{4}$ of [25], will be presented elsewhere.

\footnotetext{
${ }^{4}$ The analysis is similar to running because there are multiple phases. The problem treated here is simpler because the flight phase of running, which has three degrees of underactuation, is replaced with a fully-actuated phase.
}

\section{A. Control of the underactuated phase}

Since the stance foot toe acts as a pivot and there is no actuation at the stance foot toe, the feedback design proceeds as in [17]. Let $y_{u}=h_{u}\left(x_{u}\right)$ be a $(N-1) \times 1$ vector of output functions satisfying the following hypotheses:

HHU1) The output function $h_{u}\left(x_{u}\right)$ depends only the configuration variables;

HHU2) The decoupling matrix $L_{g_{u}} L_{f_{u}} h_{u}$ is invertible for an open set $\tilde{\mathcal{Q}}_{u} \subset \mathcal{Q}_{u}$;

HHU3) Existence of $\theta_{u}\left(q_{u}\right)$ such that $\left[h_{u}\left(q_{u}\right) ; \theta_{u}\left(q_{u}\right)\right]$ is a diffeomorphism;

HHU4) There exists a point in $\tilde{\mathcal{Q}}_{u}$ where $h_{u}$ vanishes.

HHU5) There exists a unique point $q_{u}^{-} \in \tilde{\mathcal{Q}}_{u}$ such that $\left(h_{u}\left(q_{u}^{-}\right), \Upsilon_{h}^{v}\left(q_{u}^{-}\right)\right)=(0,0), \Upsilon_{h}^{h}\left(q_{u}^{-}\right)>0$ and the rank

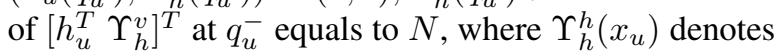
the horizontal coordinate of the swing heel.

Then there exists a smooth manifold $Z_{u}=\left\{x_{u} \in\right.$ $\left.T \mathcal{Q}_{u} \mid h_{u}\left(x_{u}\right)=0, L_{f_{u}} h_{u}\left(x_{u}\right)=0\right\}$, called the zero dynamics manifold, and $S_{u}^{f} \cap Z_{u}$ is smooth. $S_{u}^{f} \cap Z_{u}$ is one-dimensional if $S_{u}^{f} \cap Z_{u} \neq \emptyset$. Differentiating the output $y_{u}$ twice yields,

$$
\begin{aligned}
\ddot{y}_{u} & :=v_{u} \\
& =L_{f_{u}}^{2} h_{u}\left(x_{u}\right)+L_{g_{u}} L_{f_{u}} h_{u}\left(x_{u}\right) u .
\end{aligned}
$$

Since the decoupling matrix $L_{g_{u}} L_{f_{u}} h_{u}\left(x_{u}\right)$ is invertible, the feedback control $u^{*}:=$ $-\left(L_{g_{u}} L_{f_{u}} h_{u}\left(x_{u}\right)\right)^{-1}\left(L_{f_{u}}^{2} h_{u}\left(x_{u}\right)\right)$ renders the zero dynamics manifold invariant. In addition to the hypotheses HHU1-HHU5, if the hypothesis RH5 is also satisfied, then the fully-actuated phase phase zero dynamics in the coordinates of $z_{u}:=\left(\theta_{u}, \sigma_{u}\right)=\left(\theta_{u}, d_{u}\left(q_{u}\right) \dot{q}_{u}\right)$ can be written as

$$
\begin{aligned}
\dot{\theta}_{u} & =\kappa_{u}^{1}\left(\theta_{u}\right) \sigma_{u} \\
\dot{\sigma}_{u} & =\kappa_{u}^{2}\left(\sigma_{u}\right),
\end{aligned}
$$

where $u_{A}$ is the torque applied at the stance ankle, $d_{u}$ is the last row of $D_{u}$, and $\sigma_{u}$ is the angular momentum about the stance toe during the underactuated phase, [27]. Equations (11) and (12) are written as $\dot{z}_{u}=f_{Z_{u}}\left(z_{u}\right)$.

The transition map from the fully-actuated phase to the underactuated phase on the hybrid zero dynamics becomes

$$
\begin{array}{r}
\theta_{f}^{+}=\theta_{f} \circ\left[\begin{array}{cc}
R & 0
\end{array}\right] q_{u}^{-}, \\
\sigma_{f}^{+}=\delta_{u}^{f} \sigma_{u}^{-},
\end{array}
$$

where $\delta_{u}^{f}$ is a constant that can be calculated using the properties studied in [27].

\section{B. Control design for fully-actuated phase}

Since the stance foot is motionless and acting as a base during this phase, the model only has $N-1$ DOF. Consequently, the robot is fully actuated, opening up many feedback design possibilities. For example, we could, in principle, design for an empty zero dynamics, feedback linearize the model, etc. — though we would run a high 
risk of requiring so much ankle torque that the foot would rotate, thereby causing underactuation. Instead, we follow a design where, in principle, the ankle torque could be used exclusively for ensuring that the foot does not rotate, but in most cases, it can also be used to augment stability and efficiency of the overall walking cycle.

$N-2$ virtual constraints are used to create a twodimensional zero dynamics for the fully-actuated phase that is driven by the ankle torque. Let $y_{f}=h_{f}\left(x_{f}\right)$ be a $(N-2) \times 1$ vector of output functions. Let the output function $y_{f}$ satisfy the following hypotheses:

HHF1) The output function $h_{f}\left(x_{f}\right)$ depends only on the configuration variables of the fully-actuated phase;

HHF2) There exists $u_{A} \in \mathbb{R}$ such that the decoupling matrix $L_{g_{f}} L_{f_{f}^{u}} h_{f}$ is invertible for an open set $\tilde{\mathcal{Q}}_{f} \subset$ $\mathcal{Q}_{f} ;$

HHF3) There exists $\theta_{f}\left(q_{f}\right)$ such that $\left[h_{f}\left(q_{f}\right) ; \theta_{f}\left(q_{f}\right)\right]$ is diffeomorphism;

HHF4) There exists a point where $h_{f}$ vanishes;

HHF5) There exists a unique point $q_{f}^{-} \in \tilde{\mathcal{Q}}_{f}$ such that $y_{f}=h_{f}\left(q_{f}^{-}\right)=0, H_{f}^{u}\left(q_{f}^{-}\right)=0$ and $\left[h_{f} ; H_{f}^{u}\right]$ has full rank.

Then there exists a smooth manifold $Z_{f}^{u_{A}}=\left\{x_{f} \in\right.$ $\left.T \mathcal{Q}_{f} \mid h_{f}\left(x_{f}\right)=0, L_{f_{f}^{u}} h_{f}\left(x_{f}\right)=0\right\}$, called the zero dynamics manifold, and $S_{f}^{u} \cap Z_{f}$ is smooth. $S_{f}^{u} \cap Z_{f}$ is one-dimensional if $S_{f}^{u} \cap Z_{f} \neq \emptyset$.

Differentiating the output $y_{f}$ for the fully-actuated phase twice gives

$$
\begin{aligned}
\ddot{y}_{f} & :=v_{f} \\
& =L_{f_{f}^{u_{A}}}^{2} h_{f}\left(x_{f}\right)+L_{g_{f}} L_{f_{f}^{u_{A}}} h_{f}\left(x_{f}\right) u_{R} .
\end{aligned}
$$

Since $L_{g_{f}} L_{f_{f}^{u_{A}}} h_{f}$ is invertible, the feedback control $u_{R}^{*}=$ $-\left(L_{g_{f}} L_{f_{f}^{u}} h_{f}\left(x_{f}\right)\right)^{-1}\left(L_{f_{f}^{u}}^{2} h_{f}\left(x_{f}\right)\right)$ renders the zero dynamics manifold for the fully-actuated phase invariant.

In addition to the hypotheses HHF1-HHF5, if the hypothesis RH5 is also satisfied, then in the coordinates of $z_{f}:=\left(\theta_{f}, \sigma_{f}\right)=\left(\theta_{f}, d_{f}\left(q_{f}\right) \dot{q}_{f}\right)$ restricted to the zero dynamics manifold, the fully-actuated phase zero dynamics can be written as

$$
\begin{aligned}
& \dot{\theta}_{f}=\kappa_{f}^{1}\left(\theta_{f}\right) \sigma_{f} \\
& \dot{\sigma}_{f}=\kappa_{f}^{2}\left(\sigma_{f}\right)+u_{A},
\end{aligned}
$$

where $u_{A}$ is the torque applied at the stance ankle, $d_{f}$ is the last row of $D_{f}$, and $\sigma_{f}$ is the angular momentum about the stance ankle during the fully-actuated phase [27]. Equations (17) and (18) are written as $\dot{z}_{f}=f_{Z_{f}}^{u_{A}}\left(z_{f}\right)$. The transition map from the fully-actuated phase to the underactuated phase on the zero dynamics becomes

$$
\begin{array}{r}
\theta_{u}^{+}=\theta_{u} \circ\left[\begin{array}{c}
q_{f}^{-}, \\
\pi
\end{array}\right] \\
\sigma_{u}^{+}=\delta_{f}^{u} \sigma_{f}^{-},
\end{array}
$$

where $\delta_{f}^{u}$ is a constant that can be calculated using [27].

\section{Hybrid zero dynamics}

Let $Z_{f}$ be the zero dynamics manifold of the fullyactuated phase and $\dot{z}_{f}=f_{f}^{u_{A}}\left(z_{f}\right)$ be the associated zero dynamics driven by $u_{A}$. Let $\Delta_{f}^{u}$ be the transition map from the fully-actuated phase to the underactuated phase. Let $Z_{u}$ be the zero dynamics manifold of the underactuated phase and $\dot{z}_{u}=f_{u}\left(z_{u}\right)$ be the associated zero dynamics. Let $\Delta_{u}^{f}$ be the transition map from the underactuated phase to the fully-actuated phase. If $\forall z_{f} \in S_{f}^{u} \cap Z_{f}, \Delta_{f}^{u}\left(z_{f}\right) \in Z_{u}$ and $\forall z_{u} \in S_{u}^{f} \cap Z_{u}, \Delta_{u}^{f}\left(z_{u}\right) \in Z_{f}$, then

$$
\begin{cases}\dot{z}_{f}=f_{Z_{f}}^{u_{A}}\left(z_{f}\right), & z_{f}^{-} \notin S_{f}^{u} \cap Z_{f} \\ z_{u}^{+}=\Delta_{f}^{u}\left(z_{f}\right), & z_{f}^{-} \in S_{f}^{u} \cap Z_{f} \\ \dot{z}_{u}=f_{Z_{u}}\left(z_{u}\right), & z_{u}^{-} \notin S_{u}^{f} \cap Z_{u} \\ z_{f}^{+}=\Delta_{u}^{f}\left(z_{u}\right), & z_{u}^{-} \in S_{u}^{f} \cap Z_{u}\end{cases}
$$

is an invariant hybrid subsystem of the full-order hybrid model. The system (21) is called the hybrid zero dynamics and $Z_{f}$ and $Z_{u}$ are hybrid zero dynamics manifolds.

Remark 1: By definition, $Z_{f}$ and $Z_{u}$ are hybrid zero dynamics manifolds if, and only if, $\forall z_{f}^{-} \in S_{f}^{u} \cap Z_{f}$,

$$
\begin{aligned}
h_{u} \circ \Delta_{f}^{u}\left(z_{f}^{-}\right) & =0, \\
L_{f_{u}} h_{u} \circ \Delta_{f}^{u}\left(z_{f}^{-}\right) & =0,
\end{aligned}
$$

and $\forall z_{u}^{-} \in S_{u}^{f} \cap Z_{u}$ and $u_{A}=0$,

$$
\begin{aligned}
& h_{f} \circ \Delta_{u}^{f}\left(z_{u}^{-}\right)=0, \\
& L_{f_{f}^{u} A} h_{f} \circ \Delta_{u}^{f}\left(z_{u}^{-}\right)=0 .
\end{aligned}
$$

How to achieve these conditions is not developed here for reasons of space. The required conditions are a straightforward extension of [17, Sec. V].

Remark 2: Another very important result not proved here is that asymptotically stable periodic orbits of the hybrid zero dynamics are asymptotically stabilizable periodic orbits in the full-order model. The proof is based on extending the main result of [16, Thm. 2]. For an analogous result in running, see [25]. In the next section, the Poincaré map of the hybrid zero dynamics is computed in closed form.

\section{Stability Analysis on the Hybrid Zero DYNAMICS}

In general, due to the input at the stance ankle during the fully-actuated phase, the robot can move backward before it completes a step. In other words, the angular momentum about the stance ankle can be zero before entering the unactuated phase. In this paper, the angular momentum is assumed not to be zero during a step; see CH6 in Appendix A. One can think of this hypothesis as a difference between walking and dancing. Since $\sigma_{f} \neq 0$ during the fullyactuated phase, $\zeta_{f}=\frac{\sigma_{f}^{2}}{2}$ is a valid coordinate transformation. In general, $u_{A}$ can be any function of the robot's states for the zero dynamics to exist. In this paper, for simplicity, 
it is assumed to be a funcion of $\theta_{f}$ only. Then, (17) and (18) become

$$
d \zeta_{f}=\sigma_{f} d \sigma_{f}=\frac{\kappa_{f}^{2}\left(\theta_{f}\right)}{\kappa_{f}^{1}\left(\theta_{f}\right)}+\frac{u_{A}\left(\theta_{f}\right)}{\kappa_{f}^{1}\left(\theta_{f}\right)} d \theta_{f} .
$$

Let $z_{f}^{-}=\left(\theta_{f}^{-}, \sigma_{f}^{-}\right) \in S_{f}^{u} \cap Z_{f}$ and $\theta_{f}^{+}$be defined as in (19). For $\theta_{f}^{+} \leq \theta_{f} \leq \theta_{f}^{-}$, define

$$
\begin{aligned}
V_{Z_{f}}^{u_{A}}\left(\theta_{f}\right) & =-\int_{\theta_{f}^{+}}^{\theta_{f}} \frac{\kappa_{f}^{2}(\xi)}{\kappa_{f}^{1}(\xi)}+\frac{u_{A}(\xi)}{\kappa_{f}^{1}(\xi)} d \xi, \\
V_{Z_{f}}^{u_{A}, \max } & =\max _{\theta_{f}^{+} \leq \theta_{f} \leq \theta_{f}^{-}} V_{Z_{f}}^{u_{A}}\left(\theta_{f}\right) .
\end{aligned}
$$

If $\left(\delta_{f}^{u}\right)^{2} \zeta_{f}^{-}-V_{Z_{f}}^{u_{A}, \max }>0$, then (26) can be integrated, which results in

$$
\frac{1}{2}\left(\sigma_{f}^{-}\right)^{2}-\frac{1}{2}\left(\sigma_{f}^{+}\right)^{2}=\zeta_{f}^{-}-\zeta_{f}^{+}=-V_{Z_{f}}^{u_{A}}\left(\theta_{f}^{-}\right) .
$$

With (14), the Poincaré map for the fully-actuated phase $\rho_{f}: S_{u}^{f} \rightarrow S_{f}^{u}$ on the hybrid zero dynamics is defined as

$$
\rho_{f}\left(\zeta_{u}^{-}\right)=\left(\delta_{u}^{f}\right)^{2} \zeta_{u}^{-}-V_{Z_{f}}^{u_{A}}\left(\theta_{f}^{-}\right) .
$$

For the underactuated phase, the zero dynamics is equivalent to the robot with unactuated point feet, [17]. Let $z_{u}^{-}=\left(\theta_{u}^{-}, \sigma_{u}^{-}\right) \in S_{u}^{f} \cap Z_{u}$ and let $\theta_{u}^{+}$be defined as in (13). Following the procedure in [17] with (11) and (12), if $\left(\delta_{u}^{f}\right)^{2} \zeta_{u}^{-}-V_{Z_{u}}^{\max }>0$, then

$$
\frac{1}{2}\left(\sigma_{u}^{-}\right)^{2}-\frac{1}{2}\left(\sigma_{u}^{+}\right)^{2}=\zeta_{u}^{-}-\zeta_{u}^{+}=-V_{Z_{u}}\left(\theta_{u}^{-}\right),
$$

where

$$
\begin{aligned}
V_{Z_{u}}\left(\theta_{u}\right) & =-\int_{\theta_{u}^{+}}^{\theta_{u}} \frac{\kappa_{u}^{2}(\xi)}{\kappa_{u}^{1}(\xi)} d \xi, \\
V_{Z_{u}}^{\max } & =\max _{\theta_{u}^{+} \leq \theta_{u} \leq \theta_{u}^{-}} V_{Z_{u}}\left(\theta_{u}\right)
\end{aligned}
$$

The Poincaré map for the underactuated phase $\rho_{u}: S_{f}^{u} \rightarrow$ $S_{u}^{f}$ on the hybrid zero dynamics is defined with (14) as follows.

$$
\rho_{u}\left(\zeta_{f}^{-}\right)=\left(\delta_{f}^{u}\right)^{2} \zeta_{f}^{-}-V_{Z_{u}}\left(\theta_{u}^{-}\right) .
$$

Hence, the Poincaré map for the overall reduced system in $\left(\theta_{u}, \zeta_{u}\right)$ coordinates, $\rho\left(\zeta_{u}^{-}\right): S_{u}^{f} \cap Z_{u} \rightarrow S_{u}^{f} \cap Z_{u}$, is defined as follows

$$
\begin{aligned}
\rho\left(\zeta_{u}^{-}\right) & =\rho_{u} \circ \rho_{f}\left(\zeta_{u}^{-}\right) \\
& =\left(\delta_{f}^{u}\right)^{2}\left(\delta_{u}^{f}\right)^{2} \zeta_{u}^{-}-\left(\delta_{f}^{u}\right)^{2} V_{Z_{f}}^{u_{a b s}}\left(\theta_{f}^{-}\right)-V_{Z_{u}}\left(\theta_{u}^{-}\right)
\end{aligned}
$$

with domain of definition

$$
\begin{gathered}
\mathcal{D}=\left\{\zeta_{u}^{-}>0 \mid\left(\delta_{u}^{f}\right)^{2} \zeta_{u}^{-}-V_{Z_{f}}^{u_{A}, \max }>0\right. \\
\left.\left(\delta_{f}^{u}\right)^{2}\left(\delta_{u}^{f}\right)^{2} \zeta_{u}^{-}-\left(\delta_{f}^{u}\right)^{2} V_{Z_{f}}^{u_{A}}\left(\theta_{f}^{-}\right)-V_{Z_{u}}^{\max }\left(\theta_{u}^{-}\right)>0\right\} .
\end{gathered}
$$

Theorem 1: Under the hypotheses RH1-RH5, GH1GH6, and IH1-IH7 in Appendix A, HHF1-HHF5, and HHU1-HHU5,

$$
\zeta_{u}^{*}=-\frac{\left(\delta_{f}^{u}\right)^{2} V_{Z_{f}}^{u_{A}}\left(\theta_{f}^{-}\right)+V_{Z_{u}}\left(\theta_{u}^{-}\right)}{1-\left(\delta_{f}^{u}\right)^{2}\left(\delta_{u}^{f}\right)^{2}}
$$

is an exponentially stable fixed point of (35) if, and only if,

$$
\begin{aligned}
& 0<\left(\delta_{f}^{u}\right)^{2}\left(\delta_{u}^{f}\right)^{2}<1, \\
& \frac{\left(\delta_{f}^{u}\right)^{2}\left(\delta_{u}^{f}\right)^{2} V_{Z_{u}}+\left(\delta_{f}^{u}\right)^{2} V_{Z_{f}}^{u_{A}}}{1-\left(\delta_{f}^{u}\right)^{2}\left(\delta_{u}^{f}\right)^{2}}+V_{Z_{u}}^{\max }<0, \\
& \frac{\left(\delta_{f}^{u}\right)^{2}\left(\delta_{u}^{f}\right)^{2} V_{Z_{f}}^{u}+\left(\delta_{f}^{u}\right)^{2} V_{Z_{u}}}{1-\left(\delta_{f}^{u}\right)^{2}\left(\delta_{u}^{f}\right)^{2}}+V_{Z_{f}}^{u_{A}, \max }<0 .
\end{aligned}
$$

Proof: $\mathcal{D}$ is non-empty if, and only if, $\left(\delta_{f}^{u}\right)^{2}\left(\delta_{u}^{f}\right)^{2}>0$. If there exists $\zeta_{u}^{*} \in \mathcal{D}$ satisfying $\rho\left(\zeta_{u}^{*}\right)=\left(\delta_{f}^{u}\right)^{2}\left(\delta_{u}^{f}\right)^{2} \zeta_{u}^{*}-$ $\left(\delta_{f}^{u}\right)^{2} V_{Z_{f}}^{u_{A}}\left(\theta_{f}^{-}\right)-V_{Z_{u}}\left(\theta_{u}^{-}\right)$, then $\zeta^{*}$ is an exponentially stable fixed point if, and only if, $0<\left(\delta_{f}^{u}\right)^{2}\left(\delta_{u}^{f}\right)^{2}<1$, and in this case, (37) is the value of $\zeta^{*}$. Finally, (39) and (40) are the necessary and sufficient conditions for (37) to be in $\mathcal{D}$.

Note that the stability of the reduced model is not affected by the choice of $u_{A}$ since $\delta_{f}^{u}$ does not depend on $u_{A}$. However, the fixed point $\zeta^{*}$ is affected by $u_{A}$.

Remark 3: To render these analytical results useful for feedback design, a convenient finite parametrization of the virtual constraints must be introduced as in [17, Sec. V]. This will introduce free parameters into the hybrid zero dynamics, (21). A minimum energy cost criterion can then be posed and parameter optimization applied to the hybrid zero dynamics to design a provably stable, closed-loop system with satisfied design constraints, such as walking at a prescribed average speed, the forces on the support leg lying in the allowed friction cone, and the foot rotation indicator point within the hull of the foot during the fullyactuated phase and strictly in front of the foot in the underactuated phase.

\section{Special Case}

Since the feedback design for the underactuated phase has been published and illustrated elsewhere [20], [19], we consider the special case of flat-footed walking, that is, the gait consists only of the fully-actuated phase followed by an instantaneous double support phase, in order to illustrate the role of the ankle torque in our feedback design. Specializing the calculations in Section III and IV to this case, the Poincaré map of the hybrid zero dynamics is ${ }^{5}$

$$
\rho\left(\zeta_{f}^{-}\right)=\left(\delta_{u}^{f}\right)^{2} \zeta_{f}^{-}-V_{Z_{f}}^{u_{A}}\left(\theta_{f}^{-}\right),
$$

where $V_{Z}^{u_{A}}$, the potential energy (see [19]), is given in (27). The stability theorem becomes

Corollary 1: Under the hypotheses RH1-RH5, GH1GH6, and IH1-IH7 in Appendix A, HHF1-HHF5, and HHU1-HHU5,

$$
\zeta_{f}^{*}=-\frac{V_{Z_{f}}^{u_{A}}\left(\theta_{f}^{-}\right)}{1-\left(\delta_{u}^{f}\right)^{2}}
$$

\footnotetext{
${ }^{5}$ Conceptually, we are considering an instantaneous underactuated phase so that $V_{Z_{u}}=0$ and $\delta_{f}^{u}=1$
} 
is an exponentially stable fixed point of (35) if, and only if,

$$
\begin{aligned}
& 0<\left(\delta_{u}^{f}\right)^{2}<1, \\
& \frac{\left(\delta_{u}^{f}\right)^{2} V_{Z_{f}}^{u_{A}}}{1-\left(\delta_{u}^{f}\right)^{2}}+V_{Z_{f}}^{u_{A}, \max }<0 .
\end{aligned}
$$

These conditions are the same as in [17, Th. 3] for pointfeet, with the exception that the potential energy term $V_{Z_{f}}^{u_{A}}$ can be shaped by choice of the ankle torque, $u_{A}$; see second term in (27). Different shapes of the potential energy result in different fixed points and different domains of definition of the fixed points. Figure 5 shows the potential energy $V_{Z_{f}}^{u_{A}}\left(\theta_{f}\right)$ during the fully-actuated phase with different ankle torques. The solid line is when the ankle torque is a affine function of $s_{f}$ and the dashed line is when ankle torque is identically zero.

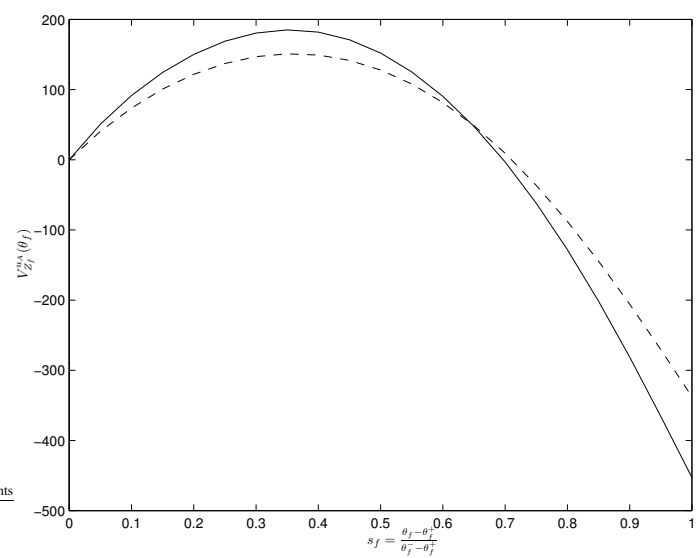

Fig. 5. Potential energies $V_{Z_{f}}^{u}$ with two different ankle inputs for a walking speed of $1.5 \mathrm{~m} / \mathrm{s}$. The potential energy can be shaped by the choice of $u_{A}$. The solid line is when $u_{A}=-21.1 s_{f}-2.9(\mathrm{Nm})$ and the dashed line is when $u_{A}=0$.

\section{CONCLUSION}

This paper has outlined a solution to the key problem of walking with both fully-actuated and underactuated phases. The studied robot was planar, bipedal, and fully actuated in the sense that it has non-trivial feet with revolute, actuated ankles and all other joints are independently actuated. The desired walking motion was assumed to consist of three successive phases: a fully-actuated phase where the stance foot is flat on the ground, an underactuated phase where the stance heel lifts from the ground and the stance foot rotates about the toe, and an instantaneous double support phase where leg exchange takes place. The main contribution of the paper was to extend the hybrid zero dynamics of [17] to a hybrid model with multiple continuous phases and varying DOF and degrees of actuation. The developed method provides a provably asymptotically stabilizing controller that integrates the fully-actuated and underactuated phases of walking. The role of the ankle torque in the proposed feedback design was emphasized by also considering the special case of a flat-footed walking gait. The ankle torque was seen as a means to directly adjust the potential energy of the hybrid zero dynamics. In this paper, the ankle-torque feedback was restricted to depend only on position, which is analogous to shaping the potential energy with a nonlinear spring. A larger class of feedbacks will be considered in future work.

\section{APPENDIX}

\section{A. Hypotheses}

The hypotheses for the robot are:

RH1) The robot consists of $\mathrm{N}$ rigid links with revolute joints;

RH2) The robot is planar;

RH3) The robot is bipedal with identical legs connected at hips;

RH4) The joints between adjacent links are actuated;

RH5) The coordinate of the robot consists of $N-1$ relative angles, $q_{1}, \cdots, q_{N-1}$, and one absolute angle, $q_{N}$.

The hypotheses for gait are:

GH1) Walking consists of three successive phases, fullyactuated phase, underactuated phase, and double support phase;

GH2) The stance foot remains on the ground during fullyactuated phase;

GH3) The stance foot does not slip during fully-actuated phase;

GH4) The stance toe acts as a pivot during underactuated phase;

GH5) The stance ankle leaves the ground without interaction;

GH6) There is no discontinuous change in positions and velocities at transition from fully-actuated phase to underactuated phase.

The hypotheses for impact are:

IH1) The swing foot has neither rebound nor slipping during impact;

IH2) After impact, the stance toe leaves the ground without any interaction with the ground;

IH3) The impact is instantaneous;

IH4) The reaction forces due to the impact can be modeled as impulses;

IH5) The impulsive forces result in discontinous changes in the velocities while the position states remain continuous;

IH6) The actuators at joints are not impulsive;

IH7) The swing ankle and the swing toe touch the ground at the same time.

The hypotheses for the closed-loop chain of double integrators, $\ddot{y}=v$, are:

$\mathrm{CH} 2$ ) Existence of solutions on $\mathbb{R}^{2 N-2}$ and uniqueness;

$\mathrm{CH} 3)$ Solutions depending continuously on the initial conditions;

CH4) The origin being globally asymptotically stable and the convergence being achieved in finite time; 
CH5) The settling time depending continuously on the initial condition;

CH6) The angular momentum about the stance ankle during the fully-actuated phase is not zero with presence of input;

CH7) The input at stance ankle during the fully-actuated phase is a function of configuration variables only.

\section{REFERENCES}

[1] H. H. R. Site, "www.honda.co.jp/robot/," January 2003.

[2] K. Hirai, M. Hirose, Y. Haikawa, and T. Takenake, "The development of Honda humanoid robot," in Proc. of the IEEE International Conference on Robotics and Automation, Leuven, Belgium, May 1998, pp. 1321-1326.

[3] S. D. S. B. E. Robot, "www.sony.net/SonyInfo/News/Press/200203/02 0319E/," January 2003.

[4] Johnnie - The TUM Biped Walking Robot, "www.amm.mw.tumuenchen.de/Misc/Messe/hanmesse-e.html,” January 2003.

[5] F. Pfeiffer, K. Loffler, and M. Gienger, "The concept of jogging johnnie," in Proc. of the IEEE International Conference on Robotics and Automation, Washington, DC, May 2002.

[6] Y. Hurmuzlu, "Dynamics of bipedal gait - part 1: objective functions and the contact event of a planar five-link biped," Journal of Applied Mechanics, vol. 60, pp. 331-336, June 1993.

[7] — - "Dynamics of bipedal gait - part 2: stability analysis of a planar five-link biped," Journal of Applied Mechanics, vol. 60, pp. 337-343, June 1993.

[8] M. Spong, "Passivity based control of the compass gait biped," in Proc. of IFAC World Congress, Beijing, China, July 1999.

[9] M. Spong and F. Bullo, "Controlled symmetries and passive walking," in Proc. of IFAC World Congress, Barcelona, Spain, July 2002.

[10] C.-L. Shih, "Ascending and descending stairs for a biped robot," IEEE Transactions on Systems, Man, and Cybernetics, vol. 29(3), pp. 255-268, May 1999.

[11] K. Yi, "Walking of a biped robot with compliant ankle joints: implementation with kubca," in Proc. of the 39th IEEE Conf. Dec. and Control, Sydney, Australia, December 2000, pp. 4809-4814.

[12] T. Takahashi and A. Kawamura, "Posture control for biped robot walk with foot toe and sole," in 27th Annual Conference of the IEEE Industrial Electronics Society, 2001, pp. 329-334.

[13] J. Park, "Impedance conrol for biped locomotion," IEEE Transactions on Robotics and Automation, vol. 17(6), pp. 870-882, December 2001.

[14] F. Silva and J. Tenreiro Machado, "Gait analysis of a human walker wearing robot feet as shoes," in Proc. of the IEEE International Conference on Robotics and Automation, Seoul, Korea, May 2001, pp. 4122-4127.

[15] M. Morisawa, Y. Fujimoto, T. Murakami, and K. Ohnishi, "A walking pattern generation for a biped robot with parallel mechanism by considering contact force," in 27th Annual Conference of the IEEE Industrial Electronics Society, 2001, pp. 2184-2189.

[16] J. Grizzle, G. Abba, and F. Plestan, "Asymptotically stable walking for biped robots: Analysis via systems with impulse effects," IEEE Transactions on Automatic Control, vol. 46, pp. 51-64, January 2001.

[17] E. Westervelt, J. Grizzle, and D. Koditschek, "Hybrid zero dynamics of planar biped walkers," IEEE Transactions on Automatic Control, vol. 48, no. 1, pp. 42-56, January 2003.

[18] E. Westervelt, G. Buche, and J. Grizzle, "Experimental validation of a framework for the design of controllers that induce stable walking in planar bipeds," The International Journal of Robotics Research, June 2004.

[19] C. Chevallereau, G. Abba, Y. Aoustin, E. Plestan, F. Westervelt, C. Canduas-de Wit, and J. Grizzle, "RABBIT: A testbed for advanced control theory," IEEE Control Systems Magazine, vol. 23, no. 5, pp. 57-79, October 2003.

[20] E. Westervelt, J. Grizzle, and C. Canudas-de-Wit, "Switching and PI control of walking motions of planar biped walkers," IEEE Transactions on Automatic Control, vol. 48, no. 2, pp. 308-312, February 2003
[21] A. D. Kuo, "Energetics of actively powered locomotion using the simplest walking model," Journal of Biomechanical Engineering, vol. 124, pp. 113-120, 2002.

[22] A. Goswami, "Postural stability of biped robots and the foot-rotation indicator (FRI) point," International Journal of Robotics Research, vol. 18, no. 6, pp. 523-533, June 1999.

[23] F. Asano, M. Yamakita, N. Kamamich, and Z.-W. Luo, "A novel gait generation for biped walking robots based on mechanical energy constraint," in Proceedings of the IEEE/RSJ Conference On Intelligent Systems and Robots, Lausanne, Switzerland, October 2002, pp. 2637-2644.

[24] Y. Hurmuzlu and D. Marghitu, "Rigid body collisions of planar kinematic chains with multiple contact points," International Journal of Robotics Research, vol. 13, no. 1, pp. 82-92, 1994.

[25] C. Chevallereau, E. Westervelt, and J. Grizzle, "Asymptotically stable running for a five-link, four-actuator, planar bipedal robot," pre-print, May 2004, submited to IJRR; copy available at available at $[28]$.

[26] J. Guckenheimer and S. Johnson, "Planar hybird systems," in Hybrid Systems II, Lecture Notes in Computer Science. Springer-Verlag, 1995, pp. 203-225.

[27] J. H. Choi and J. W. Grizzle, "Planar bipedal robot with impulsive foot action," in pre-print, 2004, submited to CDC; copy available at [28].

[28] J. Grizzle, "J.W. Grizzle's publications on robotics," 2004, www.eecs.umich.edu/ grizzle/papers/robotics.html. 